\title{
Cost Allocation for Joint Replenishment Models
}

\author{
Jiawei Zhang* \\ First Version: December 2006 \\ Revised: May 2007, July 2007
}

\begin{abstract}
We consider the one-warehouse multiple retailer inventory model with a submodular joint setup cost function. The objective of this model is to determine an inventory replenishment policy that minimizes the long-run average system cost over an infinite time horizon. Although the optimal policy for this problem is still unknown, a class of easy-to-implement power-of-two policies are $98 \%$ effective. This paper focuses on how the cost, under an optimal power-of-two policy, should be allocated to the retailers. This question generates an interesting cooperative game. We prove that this cooperative game has a non-empty core. The key to our result is a strong duality theorem for the one-warehouse multiple retailer problem under power-of-two policies.
\end{abstract}

Key Words: Cooperative Game, Joint Replenishment, Strong Duality

*Stern School of Business, IOMS-Operations Management, New York University, New York, NY, 10012. Email: jzhang@stern.nyu.edu 


\section{Introduction}

In the well-known one-warehouse multiple retailer model, constant customer demand occurs at each retailer over an infinite time horizon. The retailers place orders to the warehouse to satisfy customer demands without shortages or backlogging. These orders generate demand at the warehouse, which holds inventory and is replenished from an external supplier. For simplicity, we call the warehouse and the retailers facilities. There are two types of costs: A holding cost charged against each unit of inventory per unit time at each facility, and a setup cost charged against each order that is a submodular function of the set of facilities that places the order together. The lead times are assumed to be zero, i.e., orders are delivered instantaneously. The goal is to find an inventory replenishment policy for the system to minimize the long-run average cost over an infinite time horizon. This optimization problem and its variants have been studied extensively in the literature.

In this paper, we assume that the retailers belong to different firms. They cooperate by sharing a warehouse and by placing joint orders so as to reduce holding and setup costs. Hence, an important question arises: Once the (joint) optimization problem has been solved, how should the optimal system-wide cost be allocated among the retailers? The cost should be allocated in a way that every retailer (and every subset of retailers) would benefit from the cooperation. That is, no retailer would be better off by deviating from the coalition. Therefore, cost allocation schemes may have direct consequences on the stability of the coalition.

The cost allocation problem can be naturally modeled as a cooperative game. One solution concept of cooperative game theory, called the core, is closely related to the stability of the coalition. In what follows we study the conditions under which the cooperative game associated with the one-warehouse multiple retailer model has a non-empty core.

\subsection{Literature Review}

There has been a steadily increasing stream of research in applying cooperative game theory to problems related to inventory management. One type of cooperative game concerns inventory centralization under demand uncertainty. In this setting, a set of retailers, who 
face random demands of a single product, place a joint order before observing the demands in order to take advantage of the so-called risk-pooling effect. After the demands are realized, the inventory is optimally allocated to the retailers. It has been shown that the inventory centralization game has a non-empty core; see Hartman et al. [10] and Müller et al. [14]. More general models are studied by Slikker et al. [21], and Chen and Zhang [3].

A second type of inventory game has been studied by van den Heuvel et al. [24], Chen and Zhang [4], and Guardiola et al. [9]. The underlying optimization problem in such a game is the (deterministic) economic lot sizing (ELS) problem. In this setting, retailers can place joint orders in order to reduce setup costs. Van den Heuvel et al. [24] consider the most basic ELS game where backorders are not allowed, and show that the core is non-empty. Guardiola et al. [9] study the case when backorders are allowed, and show how to find a core allocation. Chen and Zhang [4] assume that the ordering cost is a general concave function of the order quantity, and suggest a duality approach to find a core allocation. The model studied in [4] allows backorders as well.

Our paper deals with the third type of inventory game, which concerns the cost allocation problem for an infinite time horizon joint replenishment model. In particular, this paper is closely related to Anily and Haviv [1]. Their model is essentially the same as ours with two key differences. In their model, the warehouse does not hold any inventory. Furthermore, the joint setup cost has a special structure called the first-order interaction. In this structure, a major setup cost is incurred for each order, which is independent of the set of retailers that places the order. In addition, a minor setup cost is incurred for each retailer that is included in the joint order. Yet, the optimal policy for the joint replenishment problem with first order interaction is unknown. Anily and Haviv [1] focus, therefore, on a class of easyto-implement policies, called power-of-two policies, because it is well-known that optimal power-of-two policies are 98\% effective; see Roundy [18] and Jackson et al. [11]. Anily and Haviv show that under the optimal power-of-two policy, the cooperative game associated with the joint replenishment model with first-order interaction is concave and thus has a non-empty core.

Meca et al. [13] study a special case of Anily and Haviv's model [1]. In the special case, all the minor setup costs are assumed to be zero. Therefore it is always optimal for all the retailers to order together. In a recent paper, Dror and Hartman [5] study the same model 
as Anily and Haviv [1], but their focus is mainly on characterizing a necessary and sufficient condition, under which it is optimal for all the retailers to order together and the game to have a non-empty core.

Applications of game theory in analyzing cooperations among supply chain agents can be found in Anupindi et al. [2], Granot and Sošić, [8], and Nagarajan and Sošić [15]. For an excellent comprehensive review, see Nagarajan and Sošić [16].

\subsection{Our Results and Approach}

In this paper, we show that under power-of-two policies, the joint replenishment game with a submodular joint setup cost function has a non-empty core, even when the warehouse is allowed to hold inventory. We also show that a special case of this game is concave, which generalizes one of the main results of Anily and Haviv [1].

We would like to point out that the concavity proof in [1] is tailored to the game with first order interaction. Therefore, as mentioned in [1], in order to prove the non-emptiness of the core for the joint replenishment game with a submodular joint setup cost function, new techniques have to be developed.

We take, therefore, a completely different approach, namely, a duality approach. We start by formulating the optimization problem, which aims to find the optimal power-of-two policy for the joint replenishment problem. Then we develop a Lagrangian dual of this nonconvex optimization problem. We find there is no duality gap between the Lagrangian dual and the original problem. Also, we observe that the dual variables have interesting interpretations. They indicate how the submodular joint setup cost should be divided among the retailers and the warehouse, and how the warehouse cost should be shared by the retailers. Based on these interpretations, we propose an allocation of the system-wide cost and show that the allocation is in the core of the associated cooperative game. This gives us a constructive proof of the non-emptiness of the core.

We also demonstrate that for the special case studied in [1], the corresponding Lagrangian dual problem admits a closed-form solution. This might be of interest on its own.

Duality approaches have been quite successful in analyzing cooperative games since the 
seminal work of Owen [17], who used linear programming duality to show the non-emptiness of the core for the (deterministic) linear production game. One key advantage of this duality approach is that it gives a constructive proof for the non-emptiness of the core. Although simple, the linear production game is general enough to cover a number of cooperative games such as minimum spanning tree games, flow games, discrete location games (on tree graphs), etc.; see, for instance, Tamir [22]. Thus, Owen's duality approach applies to such games as well. For the linear production game, the relationship between the allocations defined by optimal dual solutions and the core is studied by Samet and Zemel [19]. Granot [7] extends Owen's approach to a more general linear production game.

In the area of inventory management, Chen and Zhang [3] apply stochastic programming duality to a class of general inventory centralization games, and suggest a way to find coreallocations. In another paper, Chen and Zhang [4] apply linear programming duality to economic lot sizing games with concave ordering cost. In both papers [3, 4], the dual variables are interpreted as the price of the demand per unit, and the cost allocations to the retailers are proportional to their demands.

In contrast, the way we formulate the dual of the joint replenishment problem suggest a different interpretation of the dual variables. Such an interpretation is meaningful for the problem of interest, and leads to at least one of the core-allocations.

Finally, we mention that linear programming duality has been used to design polynomial time approximation algorithms for several (discrete time) inventory models; see, e.g., Levi et al. [12].

The remainder of the paper is organized as follows. In Section 2, we introduce the onewarehouse multiple retailer model and the cooperative game associated with it. In Section 3 , a strong duality theorem is developed for the one-warehouse multiple retailer problem. We then propose a class of core-allocations for the cooperative game in Section 4. Special cases of the game are studied in Section 5. We then conclude the paper and suggest areas for further study. 


\section{The Model and the Game}

In this section, we first present the infinite-horizon joint replenishment model. In this model, we are given a set of $n$ retailers, denoted by $N=\{1,2, \cdots, n\}$. The demand that retailer $i$ faces is continuous and deterministic at a fixed rate $d_{i}>0$. The retailers place orders to a single warehouse to satisfy customer demands. These orders generate demands at the warehouse, which holds inventory and is replenished from an external supplier. Backlogging is not allowed in this model. The lead time is assumed to be zero, i.e., orders arrive instantaneously.

For ease of presentation, the warehouse is denoted by 0 . Also, any $i \in N \cup\{0\}$ is called a facility, i.e., a facility can be a warehouse or a retailer.

For each $i \in N \cup\{0\}$, there is a per unit holding cost rate $h_{i}$. For simplicity we denote, $H_{i}=\frac{1}{2} h_{i} d_{i}$ and $H_{i}^{w}=\frac{1}{2} h_{0} d_{i}$, for each $i \in N$. We also assume that $0<h_{0}<h_{i}$ and thus $0<H_{i}^{w}<H_{i}$, for any $i \in N$. This assumption is common in the literature; see, e.g., Schwarz [20], Roundy [18], and Federgruen et al. [6].

When a subset $S \subseteq N \cup\{0\}$ of facilities places an order together, a joint setup cost is incurred, which is denoted by $K(S)$. Assume that

- $K(\emptyset)=0$, i.e., the joint setup cost should be zero when no facility places an order.

- $K(A) \leq K(B)$ for any $A \subseteq B \subseteq N \cup\{0\}$, i.e., the joint setup cost does not decrease when additional facilities are included in an order.

- $K(A \cup B)+K(A \cap B) \leq K(A)+K(B)$ for any $A, B \subseteq N \cup\{0\}$. It means that $K()$ is a submodular function, or equivalently, $K(A \cup\{i\})-K(A) \geq K(B \cup\{i\})-K(B)$ if $A \subseteq B$ and $i \notin A$. This property reflects general economies of scale.

We restrict ourselves to stationary inventory policies, which can be characterized by an $(N+1)$-tuple, $\left(T_{0}, T_{i}: i \in N\right)$, where $T_{0}$ is the replenishment interval at the warehouse and $T_{i}$ is that at retailer $i$ for $i=1,2, \cdots, N$. That is, facility $i \in N \cup\{0\}$ orders at times $0, T_{i}, 2 T_{i}, \cdots$. In the so-called power-of-two policy, we require that

$$
T_{i}=2^{m_{i}} \mathcal{L}, \quad \forall i \in N \cup\{0\}
$$


for some $\mathcal{L}>0$, where $m_{i}$ is an integer and can be negative. Here $\mathcal{L}$ is called the base planning period.

For an arbitrarily chosen base planning period $\mathcal{L}$, it is known from Federgruen et al. [6] that the optimal power-of-two policy yields an average cost that is at most $6 \%$ higher than the optimal cost, and thus is $94 \%$ effective. By choosing the best $\mathcal{L}$, the optimal power-of-two policy is $98 \%$ effective.

Now we consider the cost allocation problem for this joint replenishment model. We model this problem as a cooperative game denoted by $\left(N, V_{\Gamma}\right)$. Here $N$ is called the grand coalition of $n$ retailers. Any subset $S \subseteq N$ is called a coalition. $\Gamma$ is the set of all possible values that the replenishment interval $T_{i}$ can take, $i \in N \cup\{0\}$, i.e., $\Gamma$ is the set of permissible policies for every facility in $N \cup\{0\}$. If a power-of-two policy with a base planning period $\mathcal{L}$ is used, then $\Gamma$ is denoted by $\Gamma_{\mathcal{L}}$, i.e.,

$$
\Gamma_{\mathcal{L}}=\left\{t: t>0 \text { and } t=2^{m} \mathcal{L} \text { for some } m \in Z\right\}
$$

$V_{\Gamma}$ is the cost characteristic function defined for every subset of $N$, with $\Gamma$ being the set of permissible policies of every facility in $N \cup\{0\}$. In particular, $V_{\Gamma}(\emptyset)=0$ and for $\emptyset \neq S \subseteq N$, $V_{\Gamma}(S)$ is the optimal long-run average cost for the retailers in $S$.

The game $\left(N, V_{\Gamma}\right)$ is called a concave game if for every pair of subsets $S, T \subseteq N$, $V_{\Gamma}(S)+V_{\Gamma}(T) \geq V_{\Gamma}(S \cup T)+V_{\Gamma}(S \cap T)$, i.e., the set function $V_{\Gamma}(\cdot)$ is submodular.

A vector $l=\left(l_{1}, l_{2}, \cdots, l_{N}\right)$ is called an allocation for the game $\left(N, V_{\Gamma}\right)$ if $\sum_{j \in N} l_{j}=$ $V_{\Gamma}(N)$. The core of a cooperative game is a solution concept which requires that no subset of players has an incentive to secede.

Definition 1. An allocation $l$ is in the core of the game $(N, V)$, if $\sum_{j \in N} l_{j}=V_{\Gamma}(N)$ and

$$
\sum_{j \in S} l_{j} \leq V_{\Gamma}(S)
$$

for any subset $S \subseteq N$.

An allocation $l$ is called an $\epsilon$-approximate core allocation if for each subset $S \subseteq N$, $\sum_{j \in S} l_{j} \leq(1+\epsilon) V_{\Gamma}(S)$ for some $\epsilon \geq 0$. 
We now characterize the function $V_{\Gamma_{\mathcal{L}}}(S)$ for each $S \subseteq N$. Let $s=|S|$ and $\mathbb{T}_{S}=\left(T_{0}, T_{i}\right.$ : $i \in S)$. First,

$$
\begin{aligned}
V_{\Gamma_{\mathcal{L}}}(S):= & \min g_{S}\left(\mathbb{T}_{S}\right)+h_{S}\left(\mathbb{T}_{S}\right) \\
& \text { s.t. } \quad \mathbb{T}_{S} \in \Gamma_{\mathcal{L}}^{s+1},
\end{aligned}
$$

where $\Gamma_{\mathcal{L}}^{s+1}=\left\{t=\left(t_{1}, t_{2}, \cdots, t_{s+1}\right): t_{i} \in \Gamma_{\mathcal{L}}, \quad i=1,2, \cdots, s+1\right\}$. In this formulation, $g_{S}\left(\mathbb{T}_{S}\right)$ and $h_{S}\left(\mathbb{T}_{S}\right)$ are the system-wide average setup cost and inventory holding cost per unit time, respectively.

It is well-known from Roundy [18] that

$$
h_{S}\left(\mathbb{T}_{S}\right)=\sum_{i \in S}\left(H_{i} T_{i}+H_{i}^{w} \max \left\{T_{0}-T_{i}, 0\right\}\right) .
$$

We follow Federgruen et al. [6] to express $g_{S}\left(\mathbb{T}_{S}\right)$. For a given power-of-two policy specified by $\mathbb{T}_{S}$, let $\pi=\left(\pi_{0}, \pi_{1}, \cdots, \pi_{s}\right)$ be a permutation of $S \cup\{0\}$ such that

$$
T_{\pi_{0}} \leq T_{\pi_{1}} \leq \cdots \leq T_{\pi_{s}}
$$

i.e., in this permutation, the replenishment intervals are nondecreasing, and the reorder frequencies are nonincreasing. Observe that, under this power-of-two policy, whenever facility $\pi_{i}$ is replenished, all the facilities in $\left\{\pi_{0}, \cdots, \pi_{i-1}\right\}$ are replenished at the same time. Therefore, at most one of the following $s+1$ sets of facilities places an order at any replenishment epoch:

$$
\left\{\pi_{0}\right\},\left\{\pi_{0}, \pi_{1}\right\},\left\{\pi_{0}, \pi_{1}, \pi_{2}\right\}, \cdots,\left\{\pi_{0}, \pi_{1}, \cdots, \pi_{s}\right\} .
$$

Furthermore, the order frequency of the set $\left\{\pi_{0}, \pi_{1}, \cdots, \pi_{i}\right\}$ is

$$
\frac{1}{T_{\pi_{i}}}-\frac{1}{T_{\pi_{i+1}}},
$$

where $1 / T_{\pi_{s+1}}$ is defined to be zero. It follows that,

$$
\begin{aligned}
g_{S}\left(\mathbb{T}_{S}\right) & =\sum_{i=0}^{s} K\left(\left\{\pi_{0}, \cdots, \pi_{i}\right\}\right)\left(\frac{1}{T_{\pi_{i}}}-\frac{1}{T_{\pi_{i+1}}}\right) \\
& =\sum_{i=0}^{s} \frac{1}{T_{\pi_{i}}}\left(K\left(\left\{\pi_{0}, \cdots, \pi_{i-1}, \pi_{i}\right\}\right)-K\left(\left\{\pi_{0}, \cdots, \pi_{i-1}\right\}\right)\right) .
\end{aligned}
$$

Now we define

$$
\Phi_{S}=\left\{k \in R^{s+1}: k \geq 0, \quad \text { and } \quad \sum_{i \in A} k_{i} \leq K(A), \forall A \subseteq S \cup\{0\}\right\}
$$


From a well-known fact (see the definition of polymatroids and Lemma 1 in the online companion), we conclude that $g_{S}\left(\mathbb{T}_{S}\right)$ is the optimal value of the following linear program

$$
\begin{array}{ll}
\max & \sum_{i=0}^{s} \frac{1}{T_{i}} k_{i} \\
\text { s.t. } & k \in \Phi_{S} .
\end{array}
$$

Therefore, problem (1) is equivalent to

$$
V_{\Gamma_{\mathcal{L}}}(S):=\min _{\mathbb{T}_{S} \in \Gamma_{\mathcal{L}}^{s+1}} \max _{k \in \Phi_{S}} \frac{k_{0}}{T_{0}}+\sum_{i \in S}\left(\frac{k_{i}}{T_{i}}+H_{i} T_{i}+H_{i}^{w} \max \left\{T_{0}-T_{i}, 0\right\}\right) .
$$

Remark. In the cooperative game stated above, the cost function $V_{\Gamma_{\mathcal{L}}}(S)$ is defined for a given base planning period $\mathcal{L}$, which is independent of $S$. That is, all coalitions are required to use a common base planning period. This assumption is also made in Anily and Haviv [1]. Let $\left(N, V_{\Gamma_{p o t}}\right)$ denote the game in which each coalition is allowed to choose its best base planning period. On the one hand, it can be easily shown that the game $\left(N, V_{\Gamma_{p o t}}\right)$ (as well as the one studied in [1]) may have an empty core. We provide an example in Section 5 . On the other hand, any core allocation of the game $\left(N, V_{\Gamma_{\mathcal{L}}}\right)$ should be a $6 \%$-approximate core allocation for the game $\left(N, V_{\Gamma_{p o t}}\right)$. In the remainder of this paper, we focus on the game $\left(N, V_{\Gamma_{\mathcal{L}}}\right)$.

\section{Duality Results}

In this section, we develop a Lagrangian dual for problem (3). We also prove a strong duality result concerning problem (3) and its Lagrangian dual. Notice that problem (3) is defined for any subset $S \subseteq N$.

For a given $\mathbb{T}_{S}$, the dual of the linear program (2) is

$$
\begin{array}{ll}
\min _{\alpha} & \sum_{A \subseteq S \cup\{0\}} \alpha_{A} K(A) \\
\text { s.t. } & \sum_{A \subseteq S \cup\{0\}: i \in A} \alpha_{A} \geq \frac{1}{T_{i}} \quad \forall i \in S \cup\{0\} \\
& \alpha \geq 0,
\end{array}
$$


where $\alpha=\left(\alpha_{A}: A \subseteq S \cup\{0\}\right)$. Therefore, we have

$$
\begin{aligned}
V_{\Gamma_{\mathcal{L}}}(S)=\min _{\alpha, \mathbb{T}_{S}} & \sum_{A \subseteq S \cup\{0\}} \alpha_{A} K(A)+\sum_{i \in S}\left(H_{i} T_{i}+H_{i}^{w} \max \left\{T_{0}-T_{i}, 0\right\}\right) \\
\text { s.t. } & \sum_{A \subseteq S \cup\{0\}: i \in A} \alpha_{A} \geq \frac{1}{T_{i}} \quad \forall i \in S \cup\{0\} \\
& \alpha \geq 0 \\
& \mathbb{T}_{S} \in \Gamma_{\mathcal{L}}^{s+1} .
\end{aligned}
$$

We now consider a related problem where the set $\Gamma_{\mathcal{L}}$ is replaced by a more general set $\Gamma$ :

$$
\begin{array}{ll}
V_{\Gamma}(S)=\min _{\alpha, z, \mathbb{T}_{S}} & \sum_{A \subseteq S \cup\{0\}} \alpha_{A} K(A)+\sum_{i \in S}\left(H_{i} T_{i}+H_{i}^{w} z_{i}\right) \\
\text { s.t. } & \sum_{A \subseteq S \cup\{0\}: i \in A} \alpha_{A} \geq \frac{1}{T_{i}} \quad \forall i \in S \cup\{0\} \\
& z_{i} \geq T_{0}-T_{i} \quad \forall i \in S \\
& \alpha \geq 0 \\
& z \geq 0 \\
& \mathbb{T}_{S} \in \Gamma^{s+1},
\end{array}
$$

where $z=\left(z_{i}: i \in S\right)$ and $\Gamma^{s+1}=\left\{t=\left(t_{1}, t_{2}, \cdots, t_{s+1}\right): t_{i} \in \Gamma, \quad i=1,2, \cdots, s+1\right\}$. Notice that if $\Gamma=\Gamma_{\mathcal{L}}$, then the two optimization problems above are equivalent. Problem (5) is a non-convex minimization problem if the set $\Gamma$ is not convex.

Now consider a Lagrangian function related to problem (5). For each $k \in R_{+}^{s+1}$ and $u \in R_{+}^{s}$, define

$$
\begin{aligned}
& L\left(\alpha, \mathbb{T}_{S}, z ; u, k\right) \\
= & \sum_{A \subseteq S \cup\{0\}} \alpha_{A} K(A)+\sum_{i \in S}\left(H_{i} T_{i}+H_{i}^{w} z_{i}\right)-\sum_{i \in S \cup\{0\}} k_{i}\left(\sum_{A \subseteq S \cup\{0\}: i \in A} \alpha_{A}-\frac{1}{T_{i}}\right)-\sum_{i \in S} u_{i}\left(z_{i}-T_{0}+T_{i}\right) \\
= & \sum_{A \subseteq S \cup\{0\}}\left(K(A)-\sum_{i \in A} k_{i}\right) \alpha_{A}+\frac{k_{0}}{T_{0}}+\left(\sum_{i \in S} u_{i}\right) T_{0}+\sum_{i \in S}\left(\left(H_{i}-u_{i}\right) T_{i}+\frac{k_{i}}{T_{i}}\right)+\sum_{i \in S}\left(H_{i}^{w}-u_{i}\right) z_{i}
\end{aligned}
$$


Therefore, for fixed $u \geq 0$ and $k \geq 0$,

$$
\begin{aligned}
& \min _{\alpha \geq 0, z \geq 0, \mathbb{T}_{S} \in \Gamma^{s+1}} L\left(\alpha, \mathbb{T}_{S}, z ; u, k\right) \\
& =\left\{\begin{array}{l}
\min _{\mathbb{T}_{S} \in \Gamma^{s+1}} \frac{k_{0}}{T_{0}}+\left(\sum_{i \in S} u_{i}\right) T_{0}+\sum_{i \in S}\left(\left(H_{i}-u_{i}\right) T_{i}+\frac{k_{i}}{T_{i}}\right), \\
\text { if } u_{i} \leq H_{i}^{w} \quad \forall i \in S \text { and } K(A) \geq \sum_{i \in A} k_{i} \quad \forall A \subseteq S \cup\{0\} ; \\
-\infty, \quad \text { otherwise. }
\end{array}\right.
\end{aligned}
$$

It follows that the Lagrangian dual of (5) is

$$
\max _{k \in \Phi_{S}, 0 \leq u_{i} \leq H_{i}^{w}: i \in S} \min _{\mathbb{T}_{S} \in \Gamma^{s+1}} \frac{k_{0}}{T_{0}}+\left(\sum_{i \in S} u_{i}\right) T_{0}+\sum_{i \in S}\left(\left(H_{i}-u_{i}\right) T_{i}+\frac{k_{i}}{T_{i}}\right) .
$$

It turns out that the dual variables $(u, k)$ have interesting interpretations. It is clear that, for given $(u, k)$, the dual problem is completely separable, i.e., each facility just solves an EOQ problem with the power-of-two restriction. Then the variable $k$ shows how the submodular joint setup cost should be divided among the retailers and the warehouse: $k_{i}$ for retailer $i \geq 1$ and $k_{0}$ for the warehouse. Further, for each retailer $i$, the variable $u_{i}$ indicates the inventory holding cost saved by operating a warehouse. In the EOQ problem for retailer $i$, the (modified) inventory holding cost per unit inventory per unit time (multiplied by the corresponding demand) becomes $\left(H_{i}-u_{i}\right)$. On the other hand, in the EOQ problem for the warehouse, the (modified) inventory holding cost per unit inventory per unit time (multiplied by the corresponding demand) is $\sum_{i \in S} u_{i}$. These interpretations of the dual and the dual variables are particularly useful in defining core-allocations for the joint replenishment game.

It is helpful to consider an optimization problem that is closely related to problem (6):

$$
\min _{\mathbb{T}_{S} \in \Gamma^{s+1}} \max _{k \in \Phi_{S}, 0 \leq u_{i} \leq H_{i}^{w}: i \in S} \frac{k_{0}}{T_{0}}+\left(\sum_{i \in S} u_{i}\right) T_{0}+\sum_{i \in S}\left(\left(H_{i}-u_{i}\right) T_{i}+\frac{k_{i}}{T_{i}}\right) .
$$

For simplicity, define

$$
f_{S}\left(\mathbb{T}_{S} ; u, k\right)=\frac{k_{0}}{T_{0}}+\left(\sum_{i \in S} u_{i}\right) T_{0}+\sum_{i \in S}\left(\left(H_{i}-u_{i}\right) T_{i}+\frac{k_{i}}{T_{i}}\right) .
$$

The following observation is straightforward. 
Lemma 1. For any $\emptyset \neq S \subseteq N$,

$$
V_{\Gamma}(S)=\min _{\mathbb{T}_{S} \in \Gamma^{s+1}} \max _{k \in \Phi_{S}, 0 \leq u_{i} \leq H_{i}^{w}: i \in S} f_{S}\left(\mathbb{T}_{S} ; u, k\right)
$$

Proof. Recall that

$$
V_{\Gamma}(S)=\min _{\mathbb{T}_{S} \in \Gamma^{s+1}} \max _{k \in \Phi_{S}} \frac{k_{0}}{T_{0}}+\sum_{i \in S}\left(\frac{k_{i}}{T_{i}}+H_{i} T_{i}+H_{i}^{w} \max \left\{T_{0}-T_{i}, 0\right\}\right) .
$$

Therefore, the lemma follows by noticing that

$$
\sum_{i \in S}\left(T_{0}-T_{i}\right) u_{i}
$$

as a function of $u: 0 \leq u_{i} \leq H_{i}^{w}$, is maximized when

$$
u_{i}= \begin{cases}H_{i}^{w}, & \text { if } T_{0}>T_{i} \\ 0, & \text { if } T_{0}<T_{i}\end{cases}
$$

and the corresponding maximum value is $\sum_{i \in S} H_{i}^{w} \max \left\{T_{0}-T_{i}, 0\right\}$.

Lemma 1 shows that problem (7) and problem (5) have the same optimal objective value, which is equal to $V_{\Gamma}(S)$.

Our goal is to show that (7) and (6) have the same optimal objective value when $\Gamma=\Gamma_{\mathcal{L}}$. Such results usually do not hold as the set $\Gamma_{\mathcal{L}}$ is not convex. In order to establish our results, we first consider an easier case where $\Gamma=R_{+}$, which is a convex set. The following lemma is an immediate consequence of standard strong duality results in convex optimization.

Lemma 2. For any $\emptyset \neq S \subseteq N$,

$$
V_{R_{+}}(S)=\max _{k \in \Phi_{S}, 0 \leq u_{i} \leq H_{i}^{w}: i \in S} \min _{\mathbb{T}_{S} \in R_{+}^{s+1}} f_{S}\left(\mathbb{T}_{S} ; u, k\right)
$$

Proof. It is straightforward to verify that the objective function of the minimization problem (5) is convex in $\left(\mathbb{T}_{S}, \alpha, z\right)$. Recall that $\alpha=\left(\alpha_{A}: A \subseteq S \cup\{0\}\right)$ and $z=\left(z_{i}: i \in S\right)$. Furthermore, the feasible set is convex when $\Gamma=R_{+}$, and there exists a feasible solution $\left(\mathbb{T}_{S}, \alpha, z\right)$ such that all the constraints of (5) are satisfied as strict inequalities. Therefore, from Lemma 4 in the online companion, Lemma 2 follows. 
Now let $\mathbb{T}_{S}^{*}=\left(T_{0}^{*}, T_{i}^{*}: i \in S\right)$ be an optimal solution to

$$
\min _{\mathbb{T}_{S} \in R_{+}^{s+1}} \max _{k \in \Phi_{S}, 0 \leq u_{i} \leq H_{i}^{w}: i \in S} f_{S}\left(\mathbb{T}_{S} ; u, k\right)
$$

and let $\left(u^{*}, k^{*}\right)$ be an optimal solution to

$$
\max _{k \in \Phi_{S}, 0 \leq u_{i} \leq H_{i}^{w}: i \in S} \min _{\mathbb{T}_{S} \in R_{+}^{s+1}} f_{S}\left(\mathbb{T}_{S} ; u, k\right) .
$$

From Lemmas 1 and 2, we know these two optimization problems have the same optimal objective value. Therefore, it follows from Lemma 5 in the online companion that

$$
\left(u^{*}, k^{*}\right) \in \arg \max _{k \in \Phi_{S}, 0 \leq u_{i} \leq H_{i}^{w}: i \in S} f_{S}\left(\mathbb{T}_{S}^{*} ; u, k\right)
$$

and

$$
\mathbb{T}_{S}^{*} \in \arg \min _{\mathbb{T}_{S} \in R_{+}^{s+1}} f_{S}\left(\mathbb{T}_{S} ; u^{*}, k^{*}\right)
$$

From (8) and (9), we have

$$
\begin{gathered}
u_{i}^{*}=\left\{\begin{array}{l}
H_{i}^{w}, \quad \text { if } T_{0}^{*}>T_{i}^{*} \\
0, \text { if } T_{0}^{*}<T_{i}^{*},
\end{array}\right. \\
k^{*} \in \arg \max _{k \in \Phi_{S}} \sum_{i \in S \cup\{0\}} \frac{k_{i}}{T_{i}^{*}},
\end{gathered}
$$

and

$$
T_{i}^{*} \in \arg \min _{T_{i} \geq 0}\left(\frac{k_{i}^{*}}{T_{i}}+\left(H_{i}-u_{i}^{*}\right) T_{i}\right) \quad \forall i \in S .
$$

Now we are ready to prove the strong duality result concerning problem (5) and its Lagrangian dual (6) when $\Gamma=\Gamma_{\mathcal{L}}$.

Lemma 3. For any $\emptyset \neq S \subseteq N$,

$$
V_{\Gamma_{\mathcal{L}}}(S)=\max _{k \in \Phi_{S}, 0 \leq u_{i} \leq H_{i}^{w}: i \in S} \min _{\mathbb{T}_{S} \in \Gamma_{\mathcal{L}}^{s+1}} f_{S}\left(\mathbb{T}_{S} ; u, k\right)
$$

Proof. Recall that the base planning period $\mathcal{L}$ is given in the definition of $\Gamma_{\mathcal{L}}$. Assuming that $\mathbb{T}_{S}^{*}$ is given in $(9)$, we define $\tilde{\mathbb{T}}_{S}=\left(\tilde{T}_{0}, \tilde{T}_{i}: i \in S\right)$ as follows. For each $i \in S \cup\{0\}$, let $m_{i}$ be the unique integer such that

$$
2^{m_{i}-1 / 2} \mathcal{L} \leq T_{i}^{*}<2^{m_{i}+1 / 2} \mathcal{L}
$$


and let

$$
\tilde{T}_{i}=2^{m_{i}} \mathcal{L}
$$

By the definition of $\tilde{\mathbb{T}}_{S}$, we know that

$$
\tilde{T}_{0} \in \arg \min _{T_{0} \in \Gamma_{\mathcal{L}}} \frac{k_{0}^{*}}{T_{0}}+\left(\sum_{i \in S} u_{i}^{*}\right) T_{0},
$$

and

$$
\tilde{T}_{i} \in \arg \min _{T_{i} \in \Gamma_{\mathcal{L}}}\left(\frac{k_{i}^{*}}{T_{i}}+\left(H_{i}-u_{i}^{*}\right) T_{i}\right) \quad \forall i \in S .
$$

Further, for any $i, j \in S \cup\{0\}$, if $T_{i}^{*} \geq T_{j}^{*}$, then $\tilde{T}_{i} \geq \tilde{T}_{j}$; if $\tilde{T}_{i}>\tilde{T}_{j}$, then $T_{i}^{*}>T_{j}^{*}$. Thus, it follows from Lemma 3 in the online companion that

$$
\begin{gathered}
u_{i}^{*}=\left\{\begin{array}{c}
H_{i}^{w}, \quad \text { if } \tilde{T}_{0}>\tilde{T}_{i} \\
0, \quad \text { if } \tilde{T}_{0}<\tilde{T}_{i},
\end{array}\right. \\
k^{*} \in \arg \max _{k \in \Phi_{S}} \sum_{i \in S \cup\{0\}} \frac{k_{i}}{\tilde{T}_{i}} .
\end{gathered}
$$

Therefore,

$$
\left(u^{*}, k^{*}\right) \in \arg \max _{k \in \Phi_{S}, 0 \leq u_{i} \leq H_{i}^{w}: i \in S} f_{S}\left(\tilde{\mathbb{T}}_{S} ; u, k\right),
$$

and

$$
\tilde{\mathbb{T}}_{S} \in \arg \min _{\mathbb{T}_{S} \in \Gamma_{\mathcal{L}}^{s+1}} f_{S}\left(\mathbb{T}_{S} ; u^{*}, k^{*}\right),
$$

which in turn implies, in view of Lemma 5 in the online companion, that

$$
\min _{\mathbb{T}_{S} \in \Gamma_{\mathcal{L}}^{s+1}} \max _{k \in \Phi_{S}, 0 \leq u_{i} \leq H_{i}^{w}: i \in S} f_{S}\left(\mathbb{T}_{S} ; u, k\right)=\max _{k \in \Phi_{S}, 0 \leq u_{i} \leq H_{i}^{w}: i \in S} \min _{\mathbb{T}_{S} \in \Gamma_{\mathcal{L}}^{s+1}} f_{S}\left(\mathbb{T}_{S} ; u, k\right) .
$$

The proof is complete.

From our interpretation of the Lagrangian dual (6) of problem (5) and the dual variables, Lemma 3 shows that the one-warehouse multiple retailer problem under power-of-two policies is indeed separable by carefully defining cost parameters for the warehouse and the retailers. The cost parameters can be determined by an optimal dual solution $(u, k)$. 


\section{Core Allocations}

In this section, we propose an allocation to the joint replenishment game $\left(N, V_{\Gamma_{\mathcal{L}}}\right)$. We start by solving the grand dual problem of problem (3):

$$
V_{\Gamma_{\mathcal{L}}}(N)=\max _{k \in \Phi_{N}, 0 \leq u_{i} \leq H_{i}^{w}: i \in N} \min _{\mathbb{T}_{N} \in \Gamma_{\mathcal{L}}^{n+1}} f_{N}\left(\mathbb{T}_{N} ; u, k\right) .
$$

Assume that $\left(\hat{u}_{N}, \hat{k}_{N}\right)$ is an optimal solution to the grand dual problem. Further, assume that $\left(u_{N}^{*}, k_{N}^{*}\right)$ is an optimal solution to the convex relaxation of problem (18)

$$
\max _{k \in \Phi_{N}, 0 \leq u_{i} \leq H_{i}^{w}: i \in N} \min _{\mathbb{T}_{N} \in R_{+}^{n+1}} f_{N}\left(\mathbb{T}_{N} ; u, k\right) .
$$

From the proof of Lemma 3, we know that $\left(u_{N}^{*}, k_{N}^{*}\right)$ is also optimal to problem (18) (the maximization problem). Therefore, from now on, we assume $\left(\hat{u}_{N}, \hat{k}_{N}\right)=\left(u_{N}^{*}, k_{N}^{*}\right)$.

Recall the definition of $f_{N}\left(\mathbb{T}_{N} ; u, k\right)$. Given $\left(\hat{u}_{N}, \hat{k}_{N}\right), f_{N}\left(\mathbb{T}_{N} ; \hat{u}_{N}, \hat{k}_{N}\right)$ consists of the cost incurred at the warehouse and at each of the retailers. Intuitively, the cost incurred at retailer $i$ should be allocated to retailer $i$ itself. The rest of the section is devoted to allocating the cost incurred at the warehouse to the retailers.

To that end, for each $S \subseteq N$, define

$$
\gamma(S)=\min _{T_{0} \in \Gamma_{\mathcal{L}}}\left(\frac{\hat{k}_{0}}{T_{0}}+\left(\sum_{i \in S} \hat{u}_{i}\right) T_{0}\right) .
$$

The value of $\gamma(\emptyset)$ is set equal to zero. Notice that $\gamma(N)$ is the total cost incurred at the warehouse.

Now we are ready to prove our key result.

Theorem 1. Assume that a vector $\left(\theta_{i}: i \in N\right)$ satisfies

$$
\sum_{i \in N} \theta_{i}=\gamma(N)
$$

and

$$
\sum_{i \in S} \theta_{i} \leq \gamma(S) \quad \forall S \subseteq N
$$


Then the allocation $\left(\beta_{i}: i \in N\right)$ defined by

$$
\beta_{i}=\theta_{i}+\min _{T_{i} \in \Gamma_{\mathcal{L}}}\left(\frac{\hat{k}_{i}}{T_{i}}+\left(H_{i}-\hat{u}_{i}\right) T_{i}\right)
$$

is in the core of the joint replenishment game $\left(N, V_{\Gamma_{\mathcal{L}}}\right)$.

Proof. From the definition of $\left(\beta_{i}: i \in N\right)$, it is clear that,

$$
\begin{aligned}
\sum_{i \in N} \beta_{i} & =\sum_{i \in N}\left(\theta_{i}+\min _{T_{i} \in \Gamma_{\mathcal{L}}}\left(\frac{\hat{k}_{i}}{T_{i}}+\left(H_{i}-\hat{u}_{i}\right) T_{i}\right)\right) \\
& =\gamma(N)+\sum_{i \in N} \min _{T_{i} \in \Gamma_{\mathcal{L}}}\left(\frac{\hat{k}_{i}}{T_{i}}+\left(H_{i}-\hat{u}_{i}\right) T_{i}\right) \\
& =\min _{\mathbb{T}_{N} \in \Gamma_{\mathcal{L}}^{n+1}} \frac{\hat{k}_{0}}{T_{0}}+\left(\sum_{i \in N} \hat{u}_{i}\right) T_{0}+\sum_{i \in N}\left(\frac{\hat{k}_{i}}{T_{i}}+\left(H_{i}-\hat{u}_{i}\right) T_{i}\right) \\
& =\min _{k \in \Phi_{N}, 0 \leq u_{i} \leq H_{i}^{w}: i \in N} f_{\mathbb{T}_{N} \in \Gamma_{\mathcal{L}}^{n+1}}\left(\mathbb{T}_{N} ; u, k\right) \\
& =V_{\Gamma_{\mathcal{L}}}(N),
\end{aligned}
$$

where the first equality holds by the definition of $\beta_{i}$, the second holds because of assumption (19), the third holds because of the separability of the minimization problem, and the fourth holds by the definition of $\left(\hat{u}_{N}, \hat{k}_{N}\right)$.

Now, for any $S \subseteq N$, we have

$$
\begin{aligned}
\sum_{i \in S} \beta_{i} & =\sum_{i \in S}\left(\theta_{i}+\min _{T_{i} \in \Gamma_{\mathcal{L}}}\left(\frac{\hat{k}_{i}}{T_{i}}+\left(H_{i}-\hat{u}_{i}\right) T_{i}\right)\right) \\
& \leq \gamma(S)+\sum_{i \in S} \min _{T_{i} \in \Gamma_{\mathcal{L}}}\left(\frac{\hat{k}_{i}}{T_{i}}+\left(H_{i}-\hat{u}_{i}\right) T_{i}\right) \\
& =\min _{\mathbb{T}_{S} \in \Gamma_{\mathcal{L}}^{s+1}} \frac{\hat{k}_{0}}{T_{0}}+\left(\sum_{i \in S} \hat{u}_{i}\right) T_{0}+\sum_{i \in S}\left(\frac{\hat{k}_{i}}{T_{i}}+\left(H_{i}-\hat{u}_{i}\right) T_{i}\right) \\
& \leq \min _{k \in \Phi_{S}, 0 \leq u_{i} \leq H_{i}^{w}: i \in S} f_{\mathbb{T}_{S} \in \Gamma_{\mathcal{L}}^{s+1}}\left(\mathbb{T}_{S} ; u, k\right) \\
& =V_{\Gamma_{\mathcal{L}}}(S),
\end{aligned}
$$

where the first inequality holds because of assumption (20), and the second inequality holds because if $\hat{k}_{N} \in \Phi_{N}$ then $\left(\hat{k}_{0}, \hat{k}_{i}: i \in S\right) \in \Phi_{S}$. 
The core allocation $\left(\beta_{i}: i \in N\right)$ defined by (21) consists of two parts. From conditions (19) and (20), the first part $\left(\theta_{i}: i \in N\right)$ defines a (stable) allocation of the total average cost incurred at the warehouse, where the setup cost is $\hat{k}_{0}$ per order and the inventory holding cost is $\sum_{i \in S} \hat{u}_{i}$ per unit inventory and per unit time. The second term in the allocation is the average total cost for each individual retailer $i$, where the setup cost is $\hat{k}_{i}$ per order and the inventory holding cost is $\left(H_{i}-\hat{u}_{i}\right)$ per unit inventory and per unit time.

Recall that $\hat{u}_{i}=u_{i}^{*}$. From (10), it follows that if $\hat{T}_{i}>\hat{T}_{0}$ (and thus $T_{i}^{*}>T_{0}^{*}$ ), then $\hat{u}_{i}=u_{i}^{*}=0$. The implication is that, if in the optimal solution retailer $i$ orders less often than the warehouse, then the warehouse does not help retailer $i$ reduce its inventory holding cost. In this case, the (modified) inventory holding cost (multiplied by the corresponding demand) per unit time is $H_{i}-\hat{u}_{i}=H_{i}$. Also, the (modified) inventory holding cost (multiplied by the corresponding demand) per unit time, i.e., $\sum_{i \in N} \hat{u}_{i}$ does not include the contribution from retailer $i$ as $\hat{u}_{i}=0$. Therefore, it seems natural that retailer $i$ should not share the holding cost incurred at the warehouse. This observation is useful in motivating our proposed cost allocation scheme.

Notice that once $\left(\hat{u}_{N}, \hat{k}_{N}\right)=\left(u_{N}^{*}, k_{N}^{*}\right)$ is known, the minimization problem

$$
\min _{T_{i} \in \Gamma_{\mathcal{L}}}\left(\frac{\hat{k}_{i}}{T_{i}}+\left(H_{i}-\hat{u}_{i}\right) T_{i}\right)
$$

can be easily solved for each $i \in N$. Therefore, from (21), in order to find a core allocation $\left(\beta_{i}: i \in N\right)$, we only need to find a vector $\left(\theta_{i}: i \in N\right)$ that satisfies conditions (19) and (20). In the remaining part of this section, we show how this can be done.

Consider an arbitrary permutation $\left(\pi_{1}, \pi_{2}, \cdots, \pi_{n}\right)$ of $(1,2, \cdots, n)$. We define $A_{0}^{\pi}=\emptyset$, and for each $i \in N, A_{i}^{\pi}=\left\{\pi_{1}, \pi_{2}, \cdots, \pi_{i}\right\}$.

Then, for each $i \in N$, let

$$
\theta_{\pi_{i}}^{*}=\gamma\left(A_{i}^{\pi}\right)-\gamma\left(A_{i-1}^{\pi}\right)
$$

We shall show that $\left(\theta_{\pi_{i}}^{*}: i \in N\right)$ satisfies both (19) and (20) for any permutation $\left(\pi_{1}, \pi_{2}, \cdots, \pi_{n}\right)$. It is clear that (19) holds for $\left(\theta_{\pi_{i}}^{*}: i \in N\right)$. In order to verify condition (20), we first make a simple observation.

Lemma 4. The set function $\gamma(\cdot)$ is submodular. 
Proof. Consider any two sets $A \subseteq N$ and $B \subseteq N$ with non-empty intersection. Assume that there exists $T_{0}^{A} \in \Gamma_{\mathcal{L}}$ and $T_{0}^{B} \in \Gamma_{\mathcal{L}}$ such that

$$
\gamma(A)=\frac{\hat{k}_{0}}{T_{0}^{A}}+\left(\sum_{i \in A} \hat{u}_{i}\right) T_{0}^{A}
$$

and

$$
\gamma(B)=\frac{\hat{k}_{0}}{T_{0}^{B}}+\left(\sum_{i \in B} \hat{u}_{i}\right) T_{0}^{B} .
$$

Without loss of generality, we assume that $T_{0}^{A} \geq T_{0}^{B}$. If we define $T_{0}^{A \cup B}=T_{0}^{B} \in \Gamma_{\mathcal{L}}$ and $T_{0}^{A \cap B}=T_{0}^{A} \in \Gamma_{\mathcal{L}}$, then

$$
\frac{\hat{k}_{0}}{T_{0}^{A \cup B}}+\left(\sum_{i \in A \cup B} \hat{u}_{i}\right) T_{0}^{A \cup B}=\frac{\hat{k}_{0}}{T_{0}^{B}}+\left(\sum_{i \in A \backslash B} \hat{u}_{i}\right) T_{0}^{B}+\left(\sum_{i \in B} \hat{u}_{i}\right) T_{0}^{B}
$$

and

$$
\frac{\hat{k}_{0}}{T_{0}^{A \cap B}}+\left(\sum_{i \in A \cap B} \hat{u}_{i}\right) T_{0}^{A \cap B}=\frac{\hat{k}_{0}}{T_{0}^{A}}+\left(\sum_{i \in A \cap B} \hat{u}_{i}\right) T_{0}^{A} .
$$

Therefore,

$$
\begin{aligned}
& (\gamma(A \cup B)+\gamma(A \cap B))-(\gamma(A)+\gamma(B)) \\
\leq & \left(\frac{\hat{k}_{0}}{T_{0}^{A \cup B}}+\left(\sum_{i \in A \cup B} \hat{u}_{i}\right) T_{0}^{A \cup B}+\frac{\hat{k}_{0}}{T_{0}^{A \cap B}}+\left(\sum_{i \in A \cap B} \hat{u}_{i}\right) T_{0}^{A \cap B}\right)- \\
& \left(\frac{\hat{k}_{0}}{T_{0}^{A}}+\left(\sum_{i \in A} \hat{u}_{i}\right) T_{0}^{A}+\frac{\hat{k}_{0}}{T_{0}^{B}}+\left(\sum_{i \in B} \hat{u}_{i}\right) T_{0}^{B}\right) \\
= & \left(\sum_{i \in A \backslash B} \hat{u}_{i}\right)\left(T_{0}^{B}-T_{0}^{A}\right) \\
\leq & 0,
\end{aligned}
$$

where the last inequality follows from the fact that $\hat{u}_{i} \geq 0$ and $T_{0}^{B} \leq T_{0}^{A}$. This completes the proof.

Assumption (20) follows directly from Lemma 4, which is summarized in the following lemma.

Lemma 5. $\sum_{\pi_{i} \in S} \theta_{\pi_{i}}^{*} \leq \gamma(S)$ holds for any $S \subseteq N$ and for any permutation $\left(\pi_{1}, \pi_{2}, \cdots, \pi_{n}\right)$. 
Proof. We have, by the submodularity of $\gamma(\cdot)$, that

$$
\gamma\left(S \cap A_{i}^{\pi}\right)+\gamma\left(A_{i-1}^{\pi}\right) \geq \gamma\left(S \cap A_{i-1}^{\pi}\right)+\gamma\left(\left(S \cap A_{i}^{\pi}\right) \cup A_{i-1}^{\pi}\right)=\gamma\left(S \cap A_{i-1}^{\pi}\right)+\gamma\left(A_{i}^{\pi}\right)
$$

for any $S \subseteq N$ with $\pi_{i} \in S$. Thus,

$$
\gamma\left(A_{i}^{\pi}\right)-\gamma\left(A_{i-1}^{\pi}\right) \leq \gamma\left(S \cap A_{i}^{\pi}\right)-\gamma\left(S \cap A_{i-1}^{\pi}\right),
$$

which immediately implies that

$$
\sum_{\pi_{i} \in S}\left(\gamma\left(A_{i}^{\pi}\right)-\gamma\left(A_{i-1}^{\pi}\right)\right) \leq \sum_{\pi_{i} \in S}\left(\gamma\left(S \cap A_{i}^{\pi}\right)-\gamma\left(S \cap A_{i-1}^{\pi}\right)\right)=\gamma(S)
$$

The lemma follows from the definition of $\theta_{\pi_{i}}^{*}$.

Now we arrive at the main result of this section.

Corollary 1. For the joint replenishment game $\left(N, V_{\Gamma_{\mathcal{L}}}\right)$, the allocation $\left(\beta_{i}: i \in N\right)$ defined by

$$
\beta_{i}=\gamma\left(A_{i}^{\pi}\right)-\gamma\left(A_{i-1}^{\pi}\right)+\min _{T_{i} \in \Gamma_{\mathcal{L}}}\left(\frac{\hat{k}_{i}}{T_{i}}+\left(H_{i}-\hat{u}_{i}\right) T_{i}\right)
$$

is in the core for any permutation $\left(\pi_{1}, \pi_{2}, \cdots, \pi_{n}\right)$.

Notice that Corollary 1 defines a class of core-allocations. A particular allocation can be defined by choosing an arbitrary permutation $\left(\pi_{1}, \pi_{2}, \cdots, \pi_{n}\right)$. It should also be clear that any convex combination of such allocations is in the core as well. An implication of this observation is that there are infinitely many core-allocations (under very minor conditions). This fact has been proved rigorously by Anily and Haviv [1] for a special class of the game that will be discussed in the next section.

\section{$5 \quad$ Special Cases}

In this section, we consider two special cases of the general model. In the first case, the joint setup cost is additive, and the second case is the one studied by Anily and Haviv [1]. 


\subsection{Additive Setup Cost}

We consider a special case of the game $\left(N, V_{\Gamma_{\mathcal{L}}}\right)$ in which the submodular joint setup cost is additive, i.e.,

$$
K(R)=\sum_{i \in R} K_{i} \quad \forall \emptyset \neq R \subseteq N \cup\{0\},
$$

where $K_{0}$ is the setup cost of the warehouse and $K_{i}$ is the setup cost of retailer $i$.

In this case, we denote the corresponding joint replenishment game as $\left(N, V_{A \Gamma_{\mathcal{L}}}\right)$ where the characteristic cost function $V_{A \Gamma_{\mathcal{L}}}(S)$ is defined as follows, for each $S \subseteq N$ :

$$
V_{A \Gamma_{\mathcal{L}}}(S):=\min _{\mathbb{T}_{S} \in \Gamma_{\mathcal{L}}^{s+1}}\left(\frac{K_{0}}{T_{0}}+\sum_{i \in S}\left(\frac{K_{i}}{T_{i}}+H_{i} T_{i}+H_{i}^{w} \max \left(T_{0}-T_{i}, 0\right)\right)\right) .
$$

We show that the game $\left(N, V_{A \Gamma_{\mathcal{L}}}\right)$ is concave, i.e., the function $V_{A \Gamma_{\mathcal{L}}}(\cdot)$ is submodular. This fact has been proved by Teo and Shu [23], in the context of supply chain network design, for the case when $T_{0}$ and $T_{i}$ are only required to be non-negative real numbers, i.e., when the power-of-two restriction is relaxed. Nonetheless, our proof and presentation follow closely that in [23].

Theorem 2. The game $\left(N, V_{A \Gamma_{\mathcal{L}}}\right)$ is concave.

Proof. Consider any two sets $A \subseteq N$ and $B \subseteq N$ with non-empty intersection. Assume that there exist $\mathbb{T}_{A} \in \Gamma_{\mathcal{L}}^{|A|+1}$ and $\mathbb{T}_{B} \in \Gamma_{\mathcal{L}}^{|B|+1}$ such that

$$
V_{A \Gamma_{\mathcal{L}}}(A)=\frac{K_{0}}{T_{0}^{A}}+\sum_{i \in A}\left(\frac{K_{i}}{T_{i}^{A}}+H_{i} T_{i}^{A}+H_{i}^{w} \max \left(T_{0}^{A}-T_{i}^{A}, 0\right)\right)
$$

and

$$
V_{A \Gamma_{\mathcal{L}}}(B)=\frac{K_{0}}{T_{0}^{B}}+\sum_{i \in B}\left(\frac{K_{i}}{T_{i}^{B}}+H_{i} T_{i}^{B}+H_{i}^{w} \max \left(T_{0}^{B}-T_{i}^{B}, 0\right)\right) .
$$

We assume without loss of generality that $T_{0}^{A} \geq T_{0}^{B}$.

Then we define feasible solutions to problem (24) for $S=A \cup B$ and $S=A \cap B$, 
respectively as follows.

$$
\begin{aligned}
& T_{0}^{A \cup B}=T_{0}^{B} \in \Gamma_{\mathcal{L}} \\
& T_{i}^{A \cup B}=\left\{\begin{array}{lll}
T_{i}^{A} \in \Gamma_{\mathcal{L}}, & \text { if } i \in A \backslash B \\
T_{i}^{B} \in \Gamma_{\mathcal{L}}, \quad \text { if } i \in B
\end{array}\right. \\
& T_{0}^{A \cap B}=T_{0}^{A} \in \Gamma_{\mathcal{L}} \\
& T_{i}^{A \cap B}=T_{i}^{A} \in \Gamma_{\mathcal{L}} .
\end{aligned}
$$

It follows that

$$
\begin{aligned}
V_{A \Gamma_{\mathcal{L}}}(A \cup B) \leq & \frac{K_{0}}{T_{0}^{B}}+\sum_{i \in A \backslash B}\left(\frac{K_{i}}{T_{i}^{A}}+H_{i} T_{i}^{A}+H_{i}^{w} \max \left(T_{0}^{B}-T_{i}^{A}, 0\right)\right) \\
& +\sum_{i \in B}\left(\frac{K_{i}}{T_{i}^{B}}+H_{i} T_{i}^{B}+H_{i}^{w} \max \left(T_{0}^{B}-T_{i}^{B}, 0\right)\right)
\end{aligned}
$$

and

$$
V_{A \Gamma_{\mathcal{L}}}(A \cap B) \leq\left(\frac{K_{0}}{T_{0}^{A}}+\sum_{i \in A \cap B}\left(\frac{K_{i}}{T_{i}^{A}}+H_{i} T_{i}^{A}+H_{i}^{w} \max \left(T_{0}^{A}-T_{i}^{A}, 0\right)\right)\right) .
$$

Therefore,

$$
\begin{aligned}
& \left(V_{A \Gamma_{\mathcal{L}}}(A \cup B)+V_{A \Gamma_{\mathcal{L}}}(A \cap B)\right)-\left(V_{A \Gamma_{\mathcal{L}}}(A)+V_{A \Gamma_{\mathcal{L}}}(B)\right) \\
= & \sum_{i \in A \backslash B} H_{i}^{w} \max \left(T_{0}^{B}-T_{i}^{A}, 0\right)-\sum_{i \in A \backslash B} H_{i}^{w} \max \left(T_{0}^{A}-T_{i}^{A}, 0\right) \\
\leq & 0
\end{aligned}
$$

where the last inequality holds because of the assumption $T_{0}^{A} \geq T_{0}^{B}$. Thus, $V_{A \Gamma_{\mathcal{L}}}(\cdot)$ is submodular and the game $\left(N, V_{A \Gamma_{\mathcal{L}}}\right)$ is concave.

It is well-known that the core of a concave game is always non-empty. Therefore, Theorem 2 provides an alternative proof to the non-emptiness of the core when the joint setup cost is additive.

\subsection{Joint Replenishment Game with First Order Interaction}

Now we consider the game proposed and studied by Anily and Haviv [1]. In this case, the warehouse does not hold any inventory and does not place any order. For any subset 
$\emptyset \neq S \subseteq N$ of retailers, the joint setup cost is defined as

$$
K(S)=K_{0}+\sum_{i \in S} K_{i}
$$

where $K_{0}$ is called the major setup cost that is independent of set $S$, and $K_{i}$ is called the minor setup cost associated with retailer $i$. Notice that $0 \notin S$.

Under this assumption, Anily and Haviv [1] proposed a cooperative game $\left(N, V_{F \Gamma_{\mathcal{L}}}\right)$ where the characteristic function $V_{F \Gamma_{\mathcal{L}}}(S)$ is defined as follows, for each $S \subseteq N$ :

$$
\begin{aligned}
V_{F \Gamma_{\mathcal{L}}}(S)= & \min _{T_{i}: i \in S} \\
\text { s.t. } & \frac{K_{0}}{\min _{i \in S} T_{i}}+\sum_{i \in S}\left(\frac{K_{i}}{T_{i}}+H_{i} T_{i}\right) \\
& T_{i} \in \Gamma_{\mathcal{L}} \quad \forall i \in S,
\end{aligned}
$$

or equivalently

$$
\begin{array}{cl}
V_{F \Gamma_{\mathcal{L}}}(S)=\min _{\tau, T_{i}: i \in S} & \frac{K_{0}}{\tau}+\sum_{i \in S}\left(\frac{K_{i}}{T_{i}}+H_{i} T_{i}\right) \\
\text { s.t. } & T_{i} \geq \tau \quad \forall i \in S \\
& \tau, T_{i} \in \Gamma_{\mathcal{L}} \quad \forall i \in S .
\end{array}
$$

Of course, the game $\left(N, V_{F \Gamma_{\mathcal{L}}}\right)$ is a special case of the game $\left(N, V_{\Gamma_{\mathcal{L}}}\right)$. Therefore, we may apply the duality result (Lemma 2) to study the game $\left(N, V_{F \Gamma_{\mathcal{L}}}\right)$. However, given the special structure of $V_{F \Gamma_{\mathcal{L}}}(S)$, it is possible to derive a much simpler dual formulation. In particular, in the dual formulation in Lemma 2 , we have the dual variables $\left(k_{0}, k_{i}: i \in S\right)$ in addition to $\left(u_{i}: i \in S\right)$, where $k_{i}$ can be interpreted as the allocation of the joint setup cost. In this subsection, we derive a dual for problem (25), where the dual variables $\left(k_{0}, k_{i}: i \in S\right)$ are not necessary. This new dual formulation might be of interest on its own, as it is much easier to analyze. In fact, we show that the dual formulation admits a closed form solution.

For each $\lambda=\left(\lambda_{i}: i \in S\right) \geq 0$, the Lagrangian function of problem (25) is

$$
\frac{K_{0}}{\tau}+\sum_{i \in S}\left(\frac{K_{i}}{T_{i}}+H_{i} T_{i}\right)-\sum_{i \in S} \lambda_{i}\left(T_{i}-\tau\right)=\frac{K_{0}}{\tau}+\left(\sum_{i \in S} \lambda_{i}\right) \tau+\sum_{i \in S}\left(\frac{K_{i}}{T_{i}}+\left(H_{i}-\lambda_{i}\right) T_{i}\right) .
$$

Therefore, the Lagrangian dual of $(25)$ is

$$
\max _{\lambda_{i} \geq 0: \forall i \in S} \min _{\tau, T_{i} \in \Gamma_{\mathcal{L}}: \forall i \in S} \frac{K_{0}}{\tau}+\left(\sum_{i \in S} \lambda_{i}\right) \tau+\sum_{i \in S}\left(\frac{K_{i}}{T_{i}}+\left(H_{i}-\lambda_{i}\right) T_{i}\right) .
$$


Following a proof similar to that of Lemma 2, we can show that, for any $\emptyset \neq S \subseteq N$,

$$
V_{F \Gamma_{\mathcal{L}}}(S)=\max _{\lambda_{i} \geq 0: \forall i \in S} \min _{\tau, T_{i} \in \Gamma_{\mathcal{L}}: \forall i \in S} \frac{K_{0}}{\tau}+\left(\sum_{i \in S} \lambda_{i}\right) \tau+\sum_{i \in S}\left(\frac{K_{i}}{T_{i}}+\left(H_{i}-\lambda_{i}\right) T_{i}\right) .
$$

Notice that, from the Lagrangian duality result for convex optimization problems (see Lemma $? ?)$

$$
\begin{aligned}
& \max _{\lambda_{i} \geq 0: \forall i \in N} \min _{\tau, T_{i} \geq 0: \forall i \in N} \frac{K_{0}}{\tau}+\left(\sum_{i \in N} \lambda_{i}\right) \tau+\sum_{i \in N}\left(\frac{K_{i}}{T_{i}}+\left(H_{i}-\lambda_{i}\right) T_{i}\right) \\
= & \min _{\tau, T_{i} \geq 0: \forall i \in N} \max _{\lambda_{i} \geq 0: \forall i \in N} \frac{K_{0}}{\tau}+\left(\sum_{i \in N} \lambda_{i}\right) \tau+\sum_{i \in N}\left(\frac{K_{i}}{T_{i}}+\left(H_{i}-\lambda_{i}\right) T_{i}\right) \\
= & \min _{T_{i} \geq 0: \forall i \in N} \frac{K_{0}}{\min _{i \in N} T_{i}}+\sum_{i \in N}\left(\frac{K_{i}}{T_{i}}+H_{i} T_{i}\right) .
\end{aligned}
$$

Assume that $\left(\lambda_{i}^{*}: i \in N\right)$ is an optimal solution to problem $(27)$, and $\left(\tau^{*}, T_{i}^{*}: i \in N\right)$ is an optimal solution to problem (28). Then $\left(T_{i}^{*}: i \in N\right)$ is also optimal to problem (29). Therefore, $\left(T_{i}^{*}: i \in N\right)$ can be easily computed as follows.

Assume that the retailers in $\mathrm{N}$ are ordered in such a way that

$$
\frac{K_{1}}{H_{1}} \leq \frac{K_{2}}{H_{2}} \leq \cdots \leq \frac{K_{n}}{H_{n}}
$$

Let

$$
i^{*}(N)=\max \left\{j: 1 \leq j \leq n \quad \text { such that } \frac{K_{0}+\sum_{1 \leq i \leq j} K_{i}}{\sum_{1 \leq i \leq j} H_{i}} \geq \frac{K_{j}}{H_{j}}\right\}
$$

and let $N^{0}=\left\{1, \cdots, i^{*}(N)\right\}$, which is called the minimum set of $N$. Then, from [1], we have

$$
T_{i}^{*}=\left\{\begin{array}{l}
\tau^{*}=\sqrt{\frac{K_{0}+\sum_{j \in N^{0}} K_{j}}{\sum_{j \in N^{0}} H_{j}}} \text { if } i \in N^{0} \\
\sqrt{\frac{K_{i}}{H_{i}}}, \text { otherwise. }
\end{array}\right.
$$

By definition,

$$
T_{i}^{*} \in \arg \min _{T_{i} \geq 0}\left(\frac{K_{i}}{T_{i}}+\left(H_{i}-\lambda_{i}^{*}\right) T_{i}\right) .
$$

Therefore, it should be clear that

$$
\lambda_{i}^{*}=\left\{\begin{array}{l}
0 \text { if } i \notin N^{0} \\
H_{i}-\frac{K_{i}}{\left(\tau^{*}\right)^{2}}>0 \quad \text { otherwise }
\end{array}\right.
$$


As in Lemma 2, $\left(\lambda_{i}^{*}: i \in N\right)$ should be optimal to the grand dual problem

$$
V_{F \Gamma_{\mathcal{L}}}(N)=\max _{\lambda_{i} \geq 0: \forall i \in N} \min _{\tau, T_{i} \in \Gamma_{\mathcal{L}}: \forall i \in N} \frac{K_{0}}{\tau}+\left(\sum_{i \in N} \lambda_{i}\right) \tau+\sum_{i \in N}\left(\frac{K_{i}}{T_{i}}+\left(H_{i}-\lambda_{i}\right) T_{i} .\right) .
$$

To some extent, the first part of $V_{F \Gamma_{\mathcal{L}}}(N)$ corresponds to the cost incurred because of the presence of the major setup cost. We have to allocate this cost to the retailers. Then we define, for each $S \subseteq N$,

$$
\phi(S)=\min _{\tau \in \Gamma_{\mathcal{L}}} \frac{K_{0}}{\tau}+\left(\sum_{i \in S} \lambda_{i}^{*}\right) \tau
$$

Following the proof of Corollary 1, we can show that, for every permutation $\left(\pi_{1}, \pi_{2}, \cdots, \pi_{n}\right)$, the allocation $\left(\beta_{i}: i \in N\right)$ defined by

$$
\beta_{\pi_{i}}=\phi\left(\left\{\pi_{1}, \cdots, \pi_{i}\right\}\right)-\phi\left(\left\{\pi_{1}, \cdots, \pi_{i-1}\right\}\right)+\min _{T_{i} \in \Gamma_{\mathcal{L}}}\left(\frac{K_{i}}{T_{i}}+\left(H_{i}-\lambda_{i}^{*}\right) T_{i}\right)
$$

is in the core of the game $\left(N, V_{F \Gamma_{\mathcal{L}}}\right)$.

One interesting observation is that, for $\pi_{i} \notin N^{0}, \lambda_{\pi_{i}}^{*}=0$, and thus

$$
\phi\left(\left\{\pi_{1}, \cdots, \pi_{i}\right\}\right)-\phi\left(\left\{\pi_{1}, \cdots, \pi_{i-1}\right\}\right)=0,
$$

which further implies that

$$
\beta_{\pi_{i}}=\min _{T_{i} \in \Gamma}\left(\frac{K_{i}}{T_{i}}+H_{i} T_{i}\right)
$$

The implication is that the retailers not in $N^{0}$ do not pay anything towards the major setup cost. This is consistent with the core-allocation suggested by Anily and Haviv [1].

To conclude this subsection, we consider the corresponding game in which each coalition can choose its best base planning period. Consider an example with two retailers where the major setup cost is very small compared to the minor setup costs and thus can be assumed to be zero. In this case, if each retailer can optimize its base planning period, then each of them is solving an EOQ model without restrictions. Assume that the corresponding optimal costs are $V_{1}$ and $V_{2}$, respectively. Also assume that the cost parameters are chosen so that the optimal replenishment intervals of these two retailers are not power-of-two multipliers of one another. Then for the grand coalition with two retailers, when power-of-two policies are applied, the optimal cost shall be strictly higher than $V_{1}+V_{2}$. Therefore, the core for this two-player game is empty. 


\section{Concluding Remarks}

In this paper, we study the cost allocation problem for joint replenishment models. We prove that the associated cooperative game has a non-empty core. The game generalizes the one proposed by Anily and Haviv [1] to the case when the warehouse is allowed to hold inventory and the setup cost is a general submodular function of the sets of facilities that place joint orders.

The two special cases studied in Section 5 are both concave games. An immediate question is whether the general case is also concave. The answer to this question would be of interest as concave games have some interesting properties. Also, in the core-allocations proposed in [1] as well as in the current paper, there could be free riders; that is, some retailers do not share the major setup cost. Although the allocation is still stable because it is in the core, it may be considered unfair by some retailers. One way to overcome this shortcoming is to study other solution concepts in cooperative game theory. The Shapley value might be a good choice because it is in the core of this game, and under this allocation, it seems that every retailer has to share the major setup cost. However, the Shapley value could be difficult to compute as it involves the summation of exponentially many numbers. An interesting question in this direction is whether the Shapley value can be computed in polynomial time (in the number of retailers) by taking advantage of the special structure of the game.

Finally, this paper, together with [3] and [4], demonstrate the power of duality approaches in analyzing cooperative inventory games. We expect that these approaches will find more applications in such settings.

Acknowledgements The author would like to thank two anonymous referees, an associate editor, and Izak Duenyas, the area editor, for their constructive comments. The author is also grateful to Michael Pinedo and one of the referees for their valuable suggestions that considerably improve the exposition of this paper. This work was partly supported by National Science Foundation Grant CMMI-0654116. 


\section{References}

[1] Anily, S. and Haviv, M. (2007). The Cost Allocation Problem for the First Order Interaction Joint Replenishment Model. Operations Research, 55 (2), pp. 292-302.

[2] Anupindi, R., Bassok, Y. and Zemel, E. (2001). A General Framework for the Study of Decentralized Distribution Systems. Manufacturing 8 Service Operations Management, 3(4): 349-368.

[3] Chen, X. and Zhang, J. (2006). A stochastic programming duality approach to inventory centralization games. Submitted.

[4] Chen, X. and Zhang, J. (2006). Duality approaches to economic lot sizing games. Submitted.

[5] Dror, M. and Hartman, B. (2007). Shipment Consolidation: Who pays for it and how much. Management Science, 53, pp. 78-87.

[6] Federgruen, A., M. Queyranne and Y.S. Zheng. (1992). Simple power-of-two policies are close to optimal in general class of production/distribution networks with general joint setup costs. Mathematics of Operations Research, 17, 951-963.

[7] Granot, D. (1986). A generalized linear production model: A unifying model. Mathematical Programming, 34 (2) 212-222.

[8] Granot, D. and Sošić, G. (2003). A Three Stage Model for a Decentralized Distribution System of Retailers. Operations Research, 51 (5) 771-784.

[9] Guardiola, L. A., Meca, A., and Puerto, J. (2006). Coordination in periodic review inventory situations. Submitted.

[10] Hartman, B., Dror, M. and Shaked, M. (2000). Cores of inventory centralization games. Games and Economic Behavior, 31:26-49.

[11] Jackson, P., W. Maxwell and J. Muckstadt. (1985). The joint replenishment problem with a powers-of-two restriction. IIE Transactions, 17, 25-32. 
[12] Levi, R., Roundy, R.O., and Shmoys, D.B. (2006). Primal-Dual Algorithms for Deterministic Inventory Problems. Mathemtaics of Operations Research, 31(2), 267-284.

[13] Meca, A., Timmer, J., Garcia-Jurado, I., and Borm, P. (2004). Inventory games. European Journal of Operational Research, 156, 127-139.

[14] Müller, A., Scarsini, M. and Shaked, M. (2002). The newsvendor game has a non-empty core. Games and Economic Behavior, 38, 118-126.

[15] Nagarajan, M. and Sošić, G. (2007). Stable Farsighted Coalitions in Competitive Markets. Management Science, 53 (1) 29-45.

[16] Nagarajan, M. and Sošić, G. (2006). Game-Theoretical Analysis of Cooperation Among Supply Chain Agents: Review and Extensions. European Journal of Operational Research, to appear.

[17] Owen, G. (1975). On the core of linear production games. Mathematical Programming, 9, 358-370.

[18] Roundy, R.O. (1985). 98\% effective integer-ratio lot-sizing for one warehouse multiretailer systems. Management Science, 31, 1416-1430.

[19] Samet, D. and Zemel, E. (1984). On the core and dual set of linear programming games, Mathematics of Operations Research, 9, 309-316.

[20] Schwarz, L.B. (1973). A simple continuous review deterministic one-warehouse Nretailer inventory problem. Management Science, 19(5), 555-566

[21] Slikker, M., Fransoo, J., and Wouters, M. (2005). Cooperation between multiple newsvendors with transshipments. European Journal of Operational Research, 167(2), 370380.

[22] Tamir, T. (1991). On the core of network synthesis games. Mathematical Programming, $50,123-135$.

[23] Teo, C-P. and Shu, J. (2004). Warehouse-Retailer Network Design Problem. Operations Research. 
[24] van den Heuvel, W., Borm, P. E.M., and Hamers, H. (2005). Economic Lot-Sizing Games. European Journal of Operational Research, to appear. 